\title{
Hematological, biochemical and serological predictors of NSI positive dengue patients: a laboratory practical perspective
}

\begin{abstract}
Dengue fever is the most common tropical and arthropod borne viral infection in the World. The present study aimed to highlight the importance of hematological, biochemical and serological markers correlation for early diagnosis of dengue fever with NS1 antigen for timely management. The cross sectional observational study was done on 42 out of 74 patients of clinical features of dengue over a period of one year from May 2015 to April 2016. We observed a strong correlation of pancytopenia with NS1 positive patients with deranged SGPT values.
\end{abstract}

Keywords: dengue fever; pathology, NS1 antigen, hematology
Volume 4 Issue $3-2017$

\author{
Dhiraj B Nikumbh,' Rajesh Y Thakur,' \\ Roopali D Nikumbh ${ }^{3}$ \\ 'Dept of Pathology, ACPM Medical College, Dhule, India \\ ${ }^{2}$ Dept of Pathology, SBH, GMC, Dhule, India \\ ${ }^{3}$ Dept of Anatomy, ACPM Medical College, Dhule, India
}

Correspondence: Dhiraj B Nikumbh, Professor, Dept of Pathology, JMF'S ACPM Medical College, Dhule, Maharashtra, India, Email drdhirajnikumbh@rediffmail.com

Received: February 21, 2017 | Published: April 04, 2017

\section{Introduction}

Dengue fever is the most common arthropod borne viral infection of humans in tropical and subtropical countries. ${ }^{1}$ The most common epidemic vector is female Aedes aegypti mosquito for dengue. It is a major health problem worldwide including India. The World Health Organization (WHO) estimates that more than 50 millions infection occurs every year and about two fifth of world population is at risk for this infection. ${ }^{2}$ In monsoon, there are major outbreaks of dengue fever in many parts of the India. ${ }^{1}$ There are four clinical syndromes of dengue fever namely unclassified fever, classic dengue fever, dengue hemorrhagic fever and dengue shock syndrome. The main differentials of dengue fever are malaria, leptospirosis and typhoid fever in view of similar signs and symptoms and pose a diagnostic challenge. ${ }^{3}$ The present study aimed to highlight the importance of hematological, biochemical and serological markers and to correlate them for early diagnosis of dengue fever for timely management.

\section{Material and methods}

The present study was conducted in Hi-tech Diagnostic and research center, Dhule, India over a period of one year from May 2015 to April 2016. Type of Present study was cross-sectional, observational study on 74patients of clinical cases of dengue fever. All the positive patients by Dengue Day1- rapid diagnostic test (RDT) were included in the study. Only 42patients with NS1 positive were selected and included in our study. Rest (IG M and IG G positive) were excluded from the study. RDT performed were NS1 antigen and IGM, IGG antibodies by J.Mitra and Co. Pvt Limited, WHO GMP Certified kit, India with ELISA principle. Complete blood count was done on XP -100 Sysmax Hematology analyzer with principle of impedance measurement. The smears were studied with Leishman romanowasky stain for morphology. The liver function test was done on fully automated biochemistry analyzer by EM 200 machine by Transassia. After that we correlated the hematological/biochemical and serological parameters of these NS1 positive patients and discuss the results.

\section{Results}

In the present study, total of 42 confirmed NS 1 positive patients were studied over a period of one year. In our study the males outnumbered the female patients with M:F ratio was $3: 1$. The age group ranges from 3year to 45 year with mean of 7 year. We have found 42 cases of NS 1 antigen positive patients out of 74. Rest was either IGM/IGG positive with majority of IG M Positive were excluded from the study. We correlate these NS1 positive patients with hematological and biochemical findings as shown in table no. 1 and discuss the results with other researchers and draw conclusion.

Table I Laboratory parameters of study in NSI Patients

\begin{tabular}{lll}
\hline SI. No. & Parameter & Patients \\
\hline 1 & Serology .NS 1+ & $42(100 \%)$ \\
& Hematology & \\
2 & $\begin{array}{l}\text { Pancytopenia with relative } \\
\text { lymphocytosis }\end{array}$ & $29(-69 \%)$ \\
3 & $\begin{array}{l}\text { Hematology } \\
\text { Severe thrombocytopenia }\end{array}$ & $13(31 \%)$ \\
4 & $\begin{array}{l}\text { Biochemistry } \\
\text { SGPT deranged (98 U/L) }\end{array}$ & $28(66 \%)$ \\
5 & $\begin{array}{l}\text { Biochemistry } \\
\text { SGOT deranged (56 U/L) }\end{array}$ & $16(34 \%)$ \\
\hline
\end{tabular}

\section{Discussion}

We observed that 42 out of 74patients were NS1 antigen positive and remaining 32 were IGM /IGG positive with predominance of IGM positivity (28) they were not taken into account for the study. Regarding hematological correlation of these 42 NS1 patients, we observed 29 patients showed pancytopenia $(69 \%)$ with mean value of Hb was $9.8 \mathrm{gm} / \mathrm{dl}$, TLC-3200/cumm and platelet count- $48,000 /$ cumm. Severe thrombocytopenia was noted in $31 \%$ of cases with lowest platelet count was 2000/cumm. We also noted relative lymphocytosis in these patients with atypical and plasmacytoid lymphocytes with elongated tail with basophilic feathery and tapering cytoplasm. 
Our study is comparable with Mehta RC et al. ${ }^{3}$ Showed 63\% of leucopenia with relative lymphocytosis. Mehta RC et al. ${ }^{3}$ observed $81 \%$ of thrombocytopenia. In biochemical parameters, we observed contrasting features with other researchers. We found more of SGPT (98U/L) deranged than SGOT (56U/L). Majority of the patients have higher values of SGPT (66\%). As per Shukla V et al. ${ }^{1}$ the levels of SGOT were higher than SGPT. Similar findings were noted by Babaliche $\mathrm{P}$ et al. ${ }^{4}$ Impaired hepatic enzymes leading to deranged SGPT/OT; however the exact cause is unknown. But it is hypothesized that due to damaged liver can leads to abnormal transaminase values. As per Chhina RS et al., 5 involvement of myocytes leads to more deranged SGOT than SGPT. Dengue fever has no specific treatment or vaccine currently available. The differentials were malaria, leptospirosis and typhoid fever in Indian setup and often confuse in patients of pyrexia of unknown origin (PUO). ${ }^{3}$ Hence early and rapid diagnosis is key for patient management. POU patients can be suspected for dengue by hematological and biochemical parameters like pancytopenia, thrombocytopenia and deranged SGPT/OT and started on fluids till confirmation by serological tests like Dengue Day 1(RDT) test.

\section{Conclusion}

Through the above study, we concluded that hematological markers like pancytopenia, thrombocytopenia with atypical forms of lymphocytes are the diagnostic clues for the dengue. Further, biochemical deranged levels of SGPT/OT adds information regarding damaged liver function and hemolysis. Serologically, Dengue Day 1 test for NS1 antigen is always helpful for the rapid clinical diagnosis to initiates treatment, reducing morbidity and mortality in PUO patients in Indian scenario.

\section{Acknowledgements}

None.

\section{Conflict of interest}

The author declares no conflict of interest.

\section{References}

1. Shukla V, Chandra A. A study on hepatic dysfunction in dengue. J Assoc Physicians India. 2013;61(7):460-461.

2. World health organization. The special programme for research and training in tropical diseases Dengue:guidelines for diagnosis, treatment, prevention and control. WHO; 2009.

3. Mehta RC, Goswami HM, Katata RK, et al. Importance of completed blood count and peripheral smear examination in early diagnosis of dengue patients. Journal of Infectious Diseases Letters. 2013;2(1):22-24.

4. Babaliche P, Doshi D. Catching dengue early: Clinical features and laboratory markers of dengue virus infection. $J$ Assoc Physicians India. 2015;63(5):37-41.

5. Chhina RS, Goyal O, Chhina DK, et al. Clinical features and laboratory markers of dengue virus infection. J Assoc Physicians India Dengue Bull. 2008;32:110-117. 